\title{
Role of laparoscopy as diagnostic and therapeutic tool in management of omental torsion
}

Johri V, Dhaduk V, Mushtaque N, Jain N, Reddy P. K

Dept of surgical gastroenterology \& minimal Access \& bariatric surgery, Apollo Hospital Chennai, India.

Key words: Omental torsion; primary omental torsion; secondary omental torsion

\section{Introduction}

We are presenting two cases of omental torsion. Although it is a clinically rare diagnosis but when present it poses a clinical dilemma by mimicking many other common surgical illnesses presenting as acute abdomen especially acute appendicitis. Preoperative diagnosis is also very difficult because both ultrasonography and CT scan are not very specific for making an accurate diagnosis of omental torsion.

Case 1 is a rarer type of primary omental torsion which presented with symptoms of acute appendicitis albeit of less severity but ultrasound study was suggestive of appendicular mass. Intraoperatively it was found to be omental torsion with a rather normal looking appendix.

Case 2 is a more common type of secondary torsion where omentum got adhered to the site of previous open appendectomy done 30 years ago and got torted. Preoperative CT scan was suggestive of omental mass with decreased vascularity.

\section{Case presentation}

Case 1- A 38 year old gentleman presented with history of pain in right iliac fossa (RIF) for 1 day. The patient also complained of nausea. The pain was moderate in severity without any guarding. There was no history of associated fever and vomiting. His TLC and DLC were in normal range. USG study was suggestive of appendicular mass with free fluid in pelvis. Intraoperatively haemorrhagic fluid was seen in pelvis (Fig. 1A) and gangrenous omental mass with cord like torted proximal segment (Fig. 1B) was found lying alongside a normal looking appendix. Gangrenous omental mass was resected and retrieved in endobag from left hypochondrial port. Simultaneous appendicectomy was done to eliminate future diagnostic problem. The patient made uneventful recovery and hence discharged on postoperative day 1 .

\footnotetext{
Correspondence: Johri V

E-mail: vishwas@gmail.com

Received: 24-11-2017 Accepted: 25-03-2018

(iD) http://orcid.org/0000-0002-3342-5449

DOI: http://doi.org/10.4038/sljs.v36i1.8482
}

The Sri Lanka Journal of Surgery 2018; 36(1): 39-41

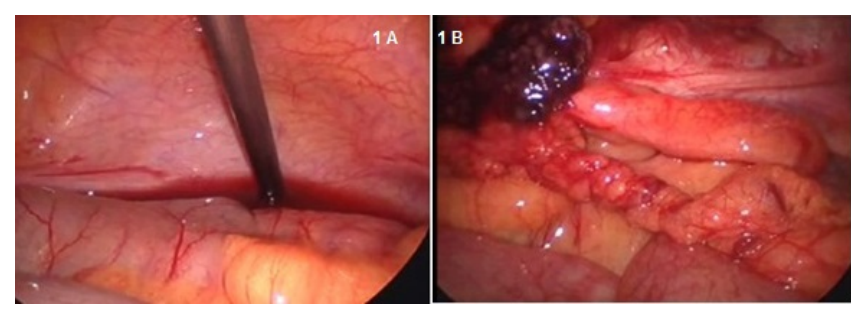

Figure 1A. Intraoperative image showing haemorrhagic fluid in pelvis. 1B. Intraoperative image showing torsion of proximal omentum and gangrenous distal omentum.

Case 2 - A 45 year old gentleman with a history of open appendicectomy done 30 years ago presented with complaints of severe pain in the RIF lasting for 3 weeks. A soft tender lump was also palpable in the RIF. It was also associated with fever and mildly raised TLC and DLC. There was no history of vomiting. CT scan revealed an omental mass with decreased vascularity adherent to anterior abdominal wall (Figure. 2A). Intraoperatively an omental mass was found in the RIF a part of which had become gangrenous. It was adherent to anterior abdominal wall, caecum and ileum (Figure. 2B). The gangrenous omentum was resected and was retrieved in endobag from left hypochondrial port. The patient made uneventful recovery and hence discharged on POD 1.

\section{Discussion}

Eitel, in 1899 first described primary omental torsion as a rare entity as a cause acute abdominal pain [1]. Since then, around 300 cases have been reported worldwide [1]. Omental torsion can be classified as "primary" and "secondary" or as "unipolar" and "bipolar".

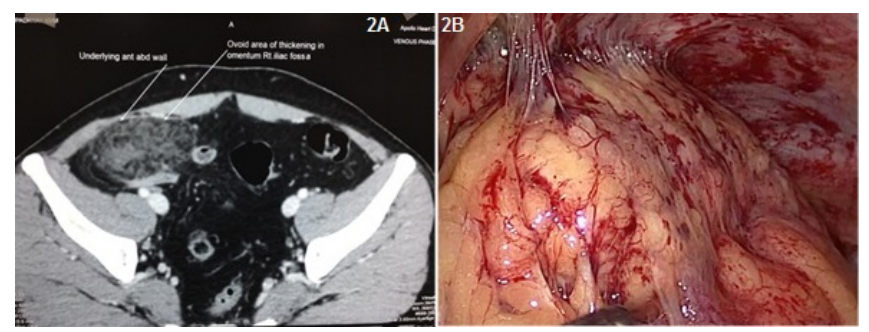

Fig. 2A. CT scan showing an omental mass adherent to anterior abdominal wall. 2B. Intraoperative image showing omental mass adherent to anterior abdominal wall at the site of previous surgery. 
Primary or idiopathic torsion occurs spontaneously without any underlying pathology and is seen less commonly. The predisposing factors for development of primary omental torsion include anatomic variations of omentum, obesity and variations in arrangement of omental blood vessels. There are conditions which may precipitate primary omental torsion in an otherwise normal omentum. These include trauma, hyperperistalsis and acute changes in body position $[1,2]$.

Secondary omental torsion is more common and is found to be associated with an underlying abdominal affection. Most commonly it is seen in association with inguinal hernias. Other conditions are omental cysts and tumours, previous abdominal surgeries, inflammatory diseases of abdomen and conditions that increase abdominal pressure [1]. In unipolar omental torsion the proximal end of omentum is fixed but distal end remains free. In bipolar omental torsion both ends of omentum are fixed [2]. Primary or idiopathic omental torsion is always unipolar but secondary omental torsion can develop both as unipolar or bipolar [2].

Clinical presentation of right sided omental torsion mimics acute appendicitis with a similar triad of pain, vomiting and temperature. The patient typically presents with a first time episode of constant, non-radiating pain in right lower quadrant which is gradually increasing in severity. It may be associated nausea and vomiting also [1]. Half of the patients develop low grade fever and leukocytosis [3]. The course of illness is generally prolonged as the patient is systemically less sick as compared to acute appendicitis. In some cases spontaneous resolution takes place and such patients may again present with another episode of torsion at a later date.

Clinical examination shows localized peritonitis. Large omental torsions may present as abdominal lumps as was seen in our patient.

Differential diagnosis in right sided omental torsion includes acute appendicitis, acute cholecystitis and twisted ovarian cyst in females. Left sided omental torsion is very uncommon and differential diagnosis includes sigmoid diverticulitis and epiploic appendagitis [1].

In radiology, ultrasonography shows complex mass with a mixture of solid and hypoechoic zones and free fluid in the peritoneal cavity. Although not very specific but it may help to rule out other more common conditions like acute appendicitis and cholecystitis. In contrast, CT scan has got high sensitivity for detecting an omental mass. The 'whirl' sign is the classical sign of omental torsion. It consists of an ill-defined omental/fatty mass with strands of twisted blood vessels whirling around a central vascular pedicle. The specificity of CT scan is low in detecting omental torsion because similar pattern is also seen in fat containing neoplasm, internal hernia containing omentocoele, epiploic appendagitis and panniculitis [4].

In selected cases, where the patient is stable and willing and diagnosis certain, conservative treatment may be offered and recovery is expected in two weeks [4]. But if the conservative trial fails it may result in formation of intra-abdominal abscess which may increase the abdominal pain, hospital stay and cost of treatment.

The laparoscopy has now become the diagnostic and therapeutic tool of choice in the management of omental torsion because low morbidity, rapid recovery and good aesthetic results that it offers. While doing diagnostic laparoscopy if one comes across a normal looking appendix and gall bladder along with haemorrhagic/serosanguinous free fluid in the peritoneal cavity (Fig. 2a), possibility of omental torsion should also be kept in mind and should be actively looked for. It is suggested that appendectomy should be done in the same sitting to avoid diagnostic dilemma in future.

\section{Conclusion}

Omental torsion is a rare entity that mimics other more common causes of acute abdomen, thereby making its primary diagnosis difficult. It should therefore be considered amongst the differential diagnosis when the primary diagnosis is doubtful. It is our suggestion that omentum should routinely be explored during laparoscopy as it provides the dual advantage of being both diagnostic as well as therapeutic tool.

All authors disclose no conflict of interest. The study was conducted in accordance with the ethical standards of the relevant institutional or national ethics committee and the Helsinki Declaration of 1975, as revised in 2000 .

\section{References}

1.Vijay Borgaonkar, Sushil Deshpande, Mukesh Rathod \& Imran Khan "Primary Omental Torsion Is a Diagnostic Challenge in Acute Abdomen - a Case Report and Literature Review" Indian J Surg (July-August 2013) 75(4):255-257

https://doi.10.1007/s12262-013-0807-6

2. Hideki Katagiri, Kunpei Honjo, Motomi Nasu, Minoru Fujisawa, and Kuniaki Kojima, "Omental Infarction due to Omental Torsion," Case Reports in Surgery, vol. 2013, Article ID 373810, 3 pages, 2013. http://dx.doi.org/10.1155/2013/373810

3. Breunung N, Strauss P A diagnostic challenge: primary omental torsion and literature review - a case report. World J Emerg Surg (2009) 4:40 https://doi.10.1186/1749-7922-4-40

4. Paresh Jain, Sheri Chhabra Omental torsion. J Indian Assoc Pediatr Surg. 2008; 13(4): 151-2.

https://doi.10.4103/0971-9261.44770 


\section{Learning Points:}

- Laparoscopy offers a unique advantage of being both diagnostic as well as a therapeutic tool when primary diagnosis is uncertain.

- In a suspected case of acute appendicitis, if a normal looking appendix is found, omental torsion should also be considered as one of the differential diagnoses. 